\section{(6) OPEN ACCESS} Seema K Shah, Department of Bioethics, National Institutes of Health, 10 Center Dr Bldg 10Rm 1C118, Bethesda 20892, Georgia, USA; shahse@mail.nih.gov

Received 30 November 2015 Revised 9 February 2016 Accepted 15 February 2016 Published Online First 3 June 2016

\section{SLinked}

- http://dx.doi.org/10.1136/ medethics-2015-103111

- http://dx.doi.org/10.1136/ medethics-2016-103416

CrossMark

To cite: Shah SK. J Med Ethics 2017;43:82-86.

\title{
When to start paediatric testing of the adult HIV cure research agenda?
}

\author{
Seema K Shah
}

\section{ABSTRACT}

Ethical guidelines recommend that experimental interventions should be tested in adults first before they are tested and approved in children. Some challenge this paradigm, however, and recommend initiating paediatric testing after preliminary safety testing in adults in certain cases. For instance, commentators have argued for accelerated testing of HIV vaccines in children. Additionally, HIV cure research on the use of very early therapy (VET) in infants, prompted in part by the Mississippi baby case, is one example of a strategy that is currently being tested in infants before it has been well tested in adults. Because infants' immune systems are still developing, the timing of HIV transmission is easier to identify in infants than in adults, and infants who receive VET might never develop the viral reservoirs that make HIV so difficult to eradicate, infants may be uniquely situated to achieve HIV cure or sustained viral remission. Several commentators have now argued for earlier initiation of HIV cure interventions other than (or in addition to) VET in children. HIV cure research is therefore a good case for re-examining the important question of when to initiate paediatric research. I will argue that, despite the potential for HIV cure research to benefit children and the scientific value of involving children in this research, the HIV cure agenda should not accelerate the involvement of children for the following reasons: HIV cure research is highly speculative, risky, aimed at combination approaches and does not compare favourably with the available alternatives. I conclude by drawing general implications for the initiation of paediatric testing, including that interventions that have to be used in combination with others and cures for chronic diseases may not be valuable enough to justify early paediatric testing.

\section{INTRODUCTION}

The prospect of finding a cure for HIV, which at times has appeared tantalisingly close to reality, has ignited public attention and raised the exciting possibility of ending the HIV/AIDS epidemic. ${ }^{1}$ Although antiretroviral therapy has effectively transformed HIV/AIDS into a chronic condition, there are still many burdens, costs, side effects and stigma facing those living with HIV/AIDS. ${ }^{2}$ This is especially true for HIV-positive children because a child, if successfully treated, will spend more time on antiretrovirals than an individual infected as an adult. Therefore, HIV research into alternatives to lifelong antiretroviral therapy, including but not limited to a cure for HIV, has great promise for adults and children.
The now famous Mississippi baby case initially seemed to offer a way to cure children with HIV by providing them with very early antiretroviral therapy (VET). In that case, an infant received antiretroviral therapy within $30 \mathrm{~h}$ of birth and was later taken off therapy for unknown reasons at approximately 18 months of life. This intervention departed from standard of care, which would require giving the infant antiretroviral drugs to prevent transmission, but with fewer drugs and at lower doses. When the Mississippi baby returned to care, replication-competent virus could not be detected in her bloodstream, and this finding could not be explained by any of the ways individuals have been able to achieve control of the virus without antiretroviral drugs in the past. $^{3}$ Unfortunately, the Mississippi baby experienced viral rebound approximately 2 years after going into remission, and was placed back on therapy in July $2014 .^{4}$

Although the Mississippi baby ultimately was not cured, the VET strategy is currently being tested in infants. This is in part because the ability to have sustained viral remission while off therapy has value on its own, and because infants may be uniquely positioned to be treated before the virus takes hold in reservoirs throughout the body that are very difficult to eliminate. Although VET is considered a cure strategy, it differs significantly from many of the strategies being used in adult cure research because VET is similar to the current standard of care, ${ }^{5}$ and the adult cure research agenda involves testing agents that are much riskier and more speculative. ${ }^{2}$ Some have argued that it is ethically defensible to test VET with certain safeguards, ${ }^{5}$ and other scholars have supplemented this analysis. ${ }^{6}$ Leaving aside VET, should other cure interventions currently being tested in adults start paediatric testing early?

There are several reasons that accelerated paediatric testing of HIV cure interventions might make sense. Some researchers argue that paediatric and adult cure agendas should 'better synergize with one another'. The scientific value of testing cure interventions on infants and children, given their unique and developing immune systems, has been cited as a reason to pursue this research as well. ${ }^{8}$ At least one HIV cure intervention-the use of stem cell transplantation from donors with an HIV-resistant mutation in patients with cancer and HIV — has already been tested in a child, though it was not successful. ${ }^{9}$ The limitations of VET have also led commentators to argue that interventions currently being tested in adults may make it easier 
to cure infants who have received VET, or that it might even be a necessary step to cure those infants. ${ }^{10}{ }^{11}$ Finally, failing to enrol children in early stages of important research can also lead to a delay in availability of the intervention down the road.

Putting children towards the front of the line for experimental cure interventions, however, flies in the face of received wisdom about the ethics of paediatric research. The general rule is to test experimental interventions in adults first. ${ }^{12}$ Some have argued that early studies of risky HIV cure interventions should not enrol patients who lack decision-making capacity, which would exclude children from the early phases of HIV cure research. ${ }^{13}$ There are some exceptions to this general rule of enrolling adults in research before children, however, which might apply to interventions that target HIV. ${ }^{14}$ This raises the question-when should the adult HIV cure agenda be translated into paediatric research?

In this paper, I first discuss the dominant view on when paediatric testing should be initiated. I then evaluate the arguments for and against HIV cure research and conclude that, despite the potential value of HIV cure interventions for children, existing experimental interventions are too risky and speculative to accelerate the involvement of children in this research, particularly in light of the available alternatives. Exceptions to this general rule are likely to be very limited. Finally, I return to the more general question of when to start testing experimental interventions in children to apply the most interesting implications from the debate over the use of HIV cure interventions in children.

\section{When should paediatric research be initiated in general?}

Children are typically defined as persons who have not attained the legal age for consent, ${ }^{15}$ and generally either parents or guardians have to provide proxy consent for children. This makes it more difficult to determine when participating in research or other activities actually reflects the values and preferences of children. Children are also more vulnerable to harm than adults, and parents or guardians may have competing interests that depart from the interests of their children, so proxy consent may be insufficient to protect children's interests in all cases. Children do develop greater decision-making capacity over time, even if they are not considered legally competent to make decisions, and can be asked for their assent. Although assent is often required by institutional review boards (IRBs) from children who have the capacity to understand some basic information about research, it does not have the same moral force as consent, and younger children are not considered capable of providing their own assent. ${ }^{16}$ Thus, providing one's own consent is a way to protect one's own interests that is precluded for most children.

In the 1970s, an important debate took place in the bioethics literature about whether it is acceptable to expose children to risk in non-beneficial research, with one prominent ethicist arguing that it is not because children do not provide their own consent, and another arguing that children can be exposed to minimal net risk in research. ${ }^{17}$ Ethicists now generally agree that children should only be exposed to relatively low amounts of net risk in research if it is justified by the benefits to society (though there is some disagreement over what counts as low risk research). ${ }^{5}$ There is also some agreement that age de-escalation (involving older children at earlier stages of research and younger children down the line) can serve as an additional protection in some cases.

Today, research with children is legally approvable by IRBs in the USA only if the risk involved is offset by a prospect of benefit, or if not, if the risk is relatively low (up to a 'minor increase over minimal risk') and if this risk is justified by the potential value for society. ${ }^{18}$ Of note, the US federal regulations also have a category in which paediatric research of higher risk and no benefit could theoretically be approved if there is sufficient social value, though there are stringent procedural hurdles, and very few studies that pose high risks have been approved in this category. ${ }^{19}$ International guidelines and regulations typically only permit paediatric research that involves minimal risk, or research that offers the potential for direct benefit. ${ }^{20}$

Controversy persists over how to interpret existing regulations and apply them to research. Weighing individual benefits against potential risk is not always straightforward. ${ }^{21}$ For instance, many scholars have questioned whether early phase research can ever be seen as beneficial to children, noting that the potential for benefit of phase I studies in particular is very low. ${ }^{22}$ Another big challenge, but one that has been much less studied, ${ }^{23}$ is determining what counts as sufficient societal benefit (often referred to as 'social value') to justify exposing children to research that poses net risk. In particular, it is hard to determine how much we should discount the social value of research based on how likely (or unlikely) it is that the research will generate a product that will help improve health. It makes intuitive sense that a very promising phase III trial that is likely to result in a product that will improve human health significantly (such as a product that has the potential to cure an otherwise fatal illness) has higher social value than a phase I trial of a similar product where the chance of failure is likely to be close to $95 \%$. Although it is often unclear how to do this type of discounting in practice, the social value of an experimental approach with a high probability of failure likely could only justify exposing individual research subjects to relatively low risk. Because social value and risk will change as a research intervention or programme moves from earlier phases to later phases, determining when children can be enrolled in research depends on the promise of any given experimental intervention as well as the phase of the research.

A European paediatric ethics working group has issued guidelines on when to initiate paediatric research that recommend the involvement of children in early phase research, including during phase II, in certain cases. This includes when the population suffers from a serious or life-threatening disease that has a different natural history than in adults, or when there is insufficient treatment for the disease. ${ }^{16}$ This analysis assumes that the interventions being tested would be beneficial to those children given the seriousness of their illness or the lack of comparable alternatives. As the analysis of HIV cure research will reveal below, however, these criteria are not sufficient for evaluating when to initiate paediatric testing.

\section{Early initiation of paediatric research on HIVIAIDS}

HIV is a serious disease that has a somewhat different course in children than in adults. In HIV prevention context, based on the scientific necessity for enrolling adolescents and the possible benefits for communities to finding an HIV vaccine, some commentators have recommended increasing the amount of risk to which adolescents can be exposed in research on an HIV vaccine, ${ }^{24}$ and enrolling older adolescents in phase IIb proof of concept HIV vaccine research. ${ }^{25}$ HIV cure research similarly has high potential benefit for society, and, as mentioned above, finding a cure in children and infants may be easier or different than finding a cure for HIV in adults. Thus, the arguments that have been made to accelerate the involvement of adolescents in HIV vaccine research would seem to apply with similar force to speed up the involvement of children in HIV cure research. However, prominent scholars have argued that children should 
not be involved in early phase HIV cure research ' $[\mathrm{b}]$ ecause the assessment of risks and benefits in these early studies is a complex and value-dependent decision'. ${ }^{15}$

This recommendation bears further examination. As mentioned above, at least one cure intervention has been tested in a child, ${ }^{7}$ and cure research has high potential for individual and societal benefit. Below I review the ethical arguments for and against earlier initiation of paediatric testing of HIV cure interventions. This analysis will reveal that, given the current state of the adult HIV cure field, it is premature to initiate paediatric testing for several reasons.

\section{Arguments in favour of involving children in early phase HIV cure research}

In theory, the prospect of a cure for HIV could be highly beneficial for children in early cure studies, as a cure would presumably allow previously HIV-infected children the chance to be healthier, live longer, be free of the burdens of daily antiretroviral therapy and to live without the stigma associated with HIV infection. As will be discussed below, however, the potential for benefit in current early phase cure research is minimal at best, what counts as a 'cure' in HIV cure research may not coincide with the traditional use of the term. The fact that alternative treatments for HIV are well established and widely available also limits the potential for benefit.

The early involvement of children in HIV cure research could have high scientific value, because the timing of infection is easier to isolate in infants who are infected in utero, during labour or during breast feeding than for people in other age groups. Children may also be more receptive to some cure interventions because their immune systems are still developing. Thus, children may be an ideal population to involve in research aiming at a cure for HIV.

Moreover, failing to involve children in early phase HIV cure research could lead to delayed access to HIV cure interventions for paediatric patients in the future. Some commentators have gone so far as to argue that '[i]t is imperative that children be included in the early stages of cure research and development, as we would not want to repeat the mistakes of the past by delaying the availability of a cure for children'. ${ }^{26}$ Such delay can occur because the translation of adult interventions into paediatric use is often far from straightforward. Significant testing may be necessary to ensure that the drugs that work in adults are safe and effective in children, and to find the right dosages, identify relevant drug-drug interactions and develop childfriendly formulations. ${ }^{27}$

Given that most early phase testing is unlikely to be successful, however, these arguments implicitly accept a trade-off between the risks to which individual research participants would be exposed and the potential for advancing scientific understanding and providing benefits for the HIV-infected children who would ultimately receive successful HIV cure interventions. As mentioned above, international regulations governing research only allow children to be exposed to relatively low net risks in research, and do not allow great sacrifices on the part of individual children to benefit others in the future. Therefore, the arguments in favour of involving children in earlier phase cure research only work insofar as early phase cure research poses relatively low risk.

\section{Arguments against involving children in early phase research}

Perhaps the most important reason to wait to enrol children in research towards an HIV cure is that the interventions being tested pose relatively high risks. HIV cure trials are investigating interventions such as chemotherapeutic agents, broadly neutralising antibodies and transplantation of autologous stem cells. ${ }^{1}$ One promising strategy is to reactivate latent reservoirs of latent T cells where HIV is lodged within the body and then destroy the virus. ${ }^{28}$ Other strategies include: 'reactivating latently infected cells and stimulating their clearance by the immune system; genetically modifying host CD4 cells to make them resistant to HIV infection'; and therapeutic vaccines. ${ }^{29}$ Key toxicities of some of the interventions being used in HIV cure research include 'immune-mediated adverse reactions, which can be severe', and 'increased risk of lymphomas, skin cancers, [and] infection'. ${ }^{26}$ These authors have noted that HIV cure interventions have risks that 'may be more pronounced in individuals with HIV' and that 'the long-term complications of many of the potentially useful agents have not been established'. ${ }^{26}$

Additionally, the research procedures needed to test the efficacy of these interventions pose significant risks. There is general agreement that 'the ultimate test of an intervention targeting the HIV-1 reservoir is an analytical treatment interruption'. ${ }^{2}$ HIV-infected individuals who interrupt their antiretroviral therapy can experience viral rebound, the seeding of reservoirs with HIV where few if any existed, the emergence of resistance to antiretrovirals to treat HIV, decreases in CD4 cell counts and unknown risks. ${ }^{5}$ It is worth noting that if interventions are found not to work in early phase research in adults, then these interventions and research methodologies will not need to be tested in children, thereby sparing them from being exposed to these risks.

A second reason not to enrol children in early phase HIV cure research is that the goal of this research is to achieve sustained viral remission, and although some possibilities have been suggested, it is not entirely clear how the ability to achieve viral remission would be useful in clinical practice. Recent experiences such as the viral rebound of the Mississippi baby have led some to conclude that 'a 'cure' for HIV infection, at least considered as a sterilizing cure with complete and definitive eradication of the virus, remains extremely challenging. Nevertheless, optimism stays high for the prospects of long-term viral suppression in the absence of ART...."30 There is an emerging consensus on the use of the term 'sustained or long-term 'remission" as the appropriate aim for HIV cure research, though commentators go on to suggest 'retaining the aspirational goal of working towards an HIV cure'. ${ }^{27}$ To be sure, sustained viral remission could be useful for children in critical developmental phases when exposure to the side effects of antiretrovirals could be especially damaging, or during periods like adolescence when adherence is often poor. ${ }^{31}$ It is still highly uncertain whether sustained viral remission is a possibility for most children, however, even with the initiation of VET. This strategy has only been tested and reported in two infants, one of whom experienced viral rebound almost immediately. Thus, the social value of research aiming at sustained viral remission is probably not sufficient to justify relatively high risks in research involving children.

Third, the different HIV cure interventions being tested are unlikely to be successful in isolation. To date, studies have not identified a single agent that can reactivate the latent HIV reservoir, and 'combination therapies with agents that potently reactivate HIV plus kill the reactivated cells will most likely be required to purge the latent reservoir in patients'. ${ }^{27}$ This strategy is sometimes referred to as 'kick and kill' or 'shock and kill'. ${ }^{26}$ As some authors have argued, 'there is clearly a long way to go and key challenges lie in the search for combination 
interventions that not only lead to activation of latent HIV infection, but also significant decreases in the size of the HIV reservoir'. ${ }^{1}$ As mentioned above, there are also several different experimental candidates for the different components of what may eventually amount to a cure. Eyal and Kuritzkes contend that the '[d]evelopment of a strategic plan to prioritise which approaches to combine will require careful thought'. ${ }^{2}$ Since cure interventions will be used in a strategy yet to be determined, the value of any individual study aiming at a cure is diminished. Unlike an HIV vaccine that might be used to prevent HIV on its own, the social value of any given cure intervention has to be divided by the amount of contribution it makes to the ultimate attainment of viral remission or cure. In other words, if one study is testing an intervention that may be a part of a five-step combination that results in viral remission, the social value of each individual intervention is a fraction of the total social value of achieving viral remission. This diminished social value makes it even less likely that testing early phase, risky HIV cure interventions could be justified in children.

Finally, the alternatives available to children living with HIV/AIDS are numerous, well tested and highly effective. Antiretroviral therapy has transformed HIV into a chronic illness, with life expectancies of those living with HIV essentially the same as people who are uninfected. Although there are side effects of antiretroviral therapy, including mitochondrial toxicity, metabolic abnormalities and skin reactions, these risks are better characterised and less severe than some of the risks associated with HIV cure interventions, and newer drugs pose fewer risks. ${ }^{32}$ Furthermore, because infants will increasingly have access to strategies for the prevention of mother to child transmission, fewer young children are likely to be infected with HIV in the future. ${ }^{33}$ Although adolescents are experiencing growing disease burden, prevention of infection is still the first line strategy, and pre-exposure prophylaxis has been shown to be highly effective for those who take it. ${ }^{34}$ Higher-income countries and lowerincome countries have also developed approaches to increase access to antiretroviral therapy around the world.

It is true, however, that many of those who are diagnosed as HIV-positive or even begin to receive care are subsequently lost to follow-up, ${ }^{35}$ and keeping patients plugged into the continuum of care is an important challenge for and a current limitation of HIV treatment programmes worldwide. Yet the cure interventions currently being tested are likely to face very similar challenges. To the extent that cure interventions are intended to result in viral remission for a period of time, people receiving these interventions will have to be closely monitored so they can be placed back on therapy when they experience viral rebound. Thus, the current challenges posed by the HIV cascade of care are unlikely to be solved even if current HIV cure interventions are successful.

In sum, current approaches to HIV cure largely do not seem to offer a radical improvement over the status quo. As Eyal and Kuritzkes have argued, cure interventions may simply 'replace safety with great uncertainty'. Without more reason to have confidence in the prospects of any given HIV cure intervention, the social value of early phase HIV cure research is simply not high enough to justify the involvement of children in this research.

\section{Limitations of the argument}

I have argued that HIV cure research should not be initiated in children until later stages of research. My argument depends on some reasonable assumptions about HIV cure research that are based on the current adult HIV cure agenda, however, and may not hold if the field changes in certain respects. For instance, if there are interventions being tested that are like VET in that they are not much riskier than the current standard of care, then this may be one exception. Additionally, if there are methodological breakthroughs that allow more careful measurement of changes in the size of viral reservoirs or better detection of very low levels of replication-competent HIV in the bloodstream, then these breakthroughs may lower the risk of some HIV cure research to a level where it makes sense to involve children in this research earlier. Another possibility, although an unlikely one, is that a cure intervention could be developed that has the potential to be used on its own, and this may also change the calculation. Finally, if a clear clinical need is articulated for children in general or a subpopulation of children to have sustained periods of time off antiretroviral therapy without incurring damage to their immune systems, initiating cure research in children may have greater value than it currently does and the earlier enrolment of children might be justifiable.

\section{CONCLUSION}

HIV cure research is currently speculative, risky, focused on combination approaches, and involves interventions that do not compare favourably with the status quo. Thus, the early involvement of children in HIV cure research is difficult to justify. There is at least one notable exception, namely research on the initiation of VET in infants, and if certain key aspects of the field achieve breakthroughs, it is possible that earlier initiation of paediatric testing of cure interventions could be justified.

This analysis has two important implications for the larger debate on when to initiate paediatric testing in comparison to adult testing. First, interventions that are likely to be part of a combination that will be used to prevent or treat an illness are less viable candidates for early initiation of paediatric testing, because the social value of each element of the combination is diminished. Second, the term 'cure' can be misleading, and for chronic conditions, a cure that amounts to sustained viral remission may not do much to improve the lives of those living with the disease. Cures for chronic diseases where lifelong treatment is available will not always be good candidates for early paediatric testing for this reason. Future work on paediatric research ethics could profit from this approach of delving deep into a field like HIV cure research to continue to refine the criteria for when it is ethically acceptable to accelerate the testing of experimental interventions in children.

Acknowledgements The author thanks Joe Millum, Sam Doernberg and Dave Wendler for helpful comments on a previous draft. The Intramural Research Program of the National Institutes of Health (NIH), the Warren G Magnuson Clinical Center, and the National Institute of Allergy and Infectious Diseases (NIAID) supported this research.

Disclaimer The opinions expressed are the views of the author. They do not represent any position or policy of the US National Institutes of Health, the Public Health Service, or the Department of Health and Human Service.

Funding The Intramural Research Program of the National Institutes of Health (NIH) Clinical Center and the National Institute of Allergy and Infectious Diseases (NIAID) supported this research.

Competing interests None declared.

Provenance and peer review Commissioned; internally peer reviewed.

Open Access This is an Open Access article distributed in accordance with the Creative Commons Attribution Non Commercial (CC BY-NC 4.0) license, which permits others to distribute, remix, adapt, build upon this work non-commercially, and license their derivative works on different terms, provided the original work is properly cited and the use is non-commercial. See: http://creativecommons.org/ licenses/by-nc/4.0/ 


\section{REFERENCES}

1 Purcell DF, Elliott JH, Ross AL, et al. Towards an HIV cure: Science and debate from the International AIDS Society 2013 symposium. Retrovirology 2013;10:134.

2 Eyal N, Kuritzkes DR. Challenges in clinical trial design for HIV-1 cure research. Lancet 2013;382:1464-5.

3 Persaud D, Gay H, Ziemniak C, et al. Absence of detectable HIV-1 viremia after treatment cessation in an infant. N Engl J Med 2013;369:1828-35.

4 Luzuriaga K, Gay H, Ziemniak C, et al. Viremic relapse after HIV-1 remission in a perinatally infected child. N Engl J Med 2015;372:786-8.

5 Shah SK, Persaud D, Wendler DS, et al. Research into a functional cure for HIV in neonates: the need for ethical foresight, Lancet Infect Dis 2014;14:893-8.

6 Rossouw TM, Crane J, Frenkel LM. Ethics of HIV research in children. Lancet Infect Dis 2015;15:634-5.

7 Soudens $\mathrm{H}$, et al. Pediatric HIV cure research in Canada (presentation); IAS 2015: Towards and HIV Cure symposium, Vancouver, Canada.

8 Klein N, Palma P, Luzuriaga K, et al. Early antiretroviral therapy in children perinatally infected with HIV: a unique opportunity to implement immunotherapeutic approaches to prolong viral remission. Lancet Infect Dis 2015;15:1108-14.

9 Paquette J. Pediatric patient dies after undergoing historic transplant at $U$ of $M$. University of Minnesota Press Release, 2013, http://www.healthtalk.umn.edu/2013/ 07/13/pediatric-patient-dies-after-undergoing-historic-transplant-at-u-of-m/

10 Pace M, Frater J. A cure for HIV: is it in sight? Expert Rev Anti Infect Ther 2014;12:783-91.

11 Rainwater-Lovett K, Luzuriaga K, Persaud D. Very early combination antiretroviral therapy in infants: prospects for cure. Curr Opinion HIV \& AIDS 2015;10:4-11.

12 Council for International Organizations of Medical Sciences (CIOMS). International ethical guidelines for biomedical research involving human subjects. Geneva: World Health Organization, 2002. http://www.cioms.ch/publications/layout_guide2002.pdf

13 Lo B, Grady C. Ethical considerations in HIV cure research: points to consider. Curr Opin HIV AIDS 2013;8:2434-9.

14 Gill D, Ethics Working Group of the Confederation of European Specialists in Paediatrics. Ethical principles and operational guidelines for good clinical practice in paediatric research. Recommendations of the Ethics Working Group of the Confederation of European Specialists in Paediatrics (CESP). Eur J Pediatr 2004;163:53-7.

1545 C.F.R. Subpart D, 46.402.
16 Whittle $A$, Shah $\mathrm{S}$, Wilfond $\mathrm{B}$, et al. Institutional review board practices regarding assent in pediatric research. Pediatrics 2004;113:1747.

17 Ramsey P. The enforcement of morals: nontherapeutic research on children. Hastings Cent Rep 1976;6:21-30.

1845 C.F.R. 46, Subpart D.

19 Wendler D, Varma S. Minimal risk in pediatric research. J Pediatr 2006;149:855-61.

20 Berglund, C A. Children in medical research: Australian ethical standards. Child Care Health Dev 1995;21:149-59.

21 Rid A, Emanuel EJ, Wendler D. Evaluating the risks of clinical research. JAMA 2010;304:1472-9.

22 Ross LF. Phase I research and the meaning of direct benefit. J Pediatr 2006;149(1 Suppl):S20-4

23 Wertheimer $A$. The social value requirement reconsidered. Bioethics 2015;29:301-8.

24 Snyder J, Miller CL, Gray G. Relative versus absolute standards for everyday risk in adolescent HIV prevention trials: expanding the debate. Am J Bioeth 2011;11:5-13.

25 Slack C, Strode A, Fleischer T, et al. Enrolling adolescents in HIV vaccine trials: reflections on legal complexities from South Africa. BMC Med Ethics 2007;8:5.

26 Lyons C, Hellman N. Setting the Stage for an HIV Cure. Huffington Post 02/03/2014.

27 Nachman S, Ahmed A, Amanullah F, et al. Towards early inclusion of children in tuberculosis drugs trials: a consensus statement. Lancet Infect Dis 2015;15:711-20.

28 Søgaard OS, Graversen ME, Leth $\mathrm{S}$, et al. The depsipeptide romidepsin reverses HIV-1 latency in vivo. PLoS Pathog 2015;11:e1005142.

29 Polizzotto MN, Chen G, Tressler RL, et al. Leveraging cancer therapeutics for the HIV cure agenda: current status and future directions. Drugs 2015;75:1447-59.

30 Anderson JL, Fromentin R, Corbelli GM, et al. Progress toward an HIV cure: update from the 2014 International AIDS Society Symposium. AIDS Res Hum Retroviruses 2015:31:36-44, at 42

31 Bernheimer JM, Patten G, Makeleni T, et al. Paediatric HIV treatment failure: a silent epidemic. J Int AIDS Soc 2015;18:20090.

32 Fernández-Monteroa JVF, Vispoa E, Soriano V. Emerging antiretroviral drugs, Expert Opin Pharmacother 2014;15:211-19.

33 WHO 2013 guidelines PMTCT.

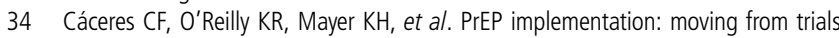
to policy and practice. J Int AIDS Soc 2015;18(Suppl 3):20222. eCollection 2015.

35 Rosen S, Fox MP. Retention in HIV care between testing and treatment in sub-Saharan Africa: a systematic review. PLoS Med 2011;8:e1001056. 